\title{
GENERALIZATION OF THE FROBENIUS THEOREM ON INVOLUTIVITY
}

\author{
CHONG-KYU HAN
}

\begin{abstract}
Given a system of $s$ independent 1-forms on a smooth manifold $M$ of dimension $m$, we study the existence of integral manifolds by means of various generalized versions of the Frobenius theorem. In particular, we present necessary and sufficient conditions for there to exist $s^{\prime}$-parameter $\left(s^{\prime}<s\right)$ family of integral manifolds of dimension $p:=m-s$, and a necessary and sufficient condition for there to exist integral manifolds of dimension $p^{\prime}, p^{\prime} \leq p$. We also present examples and applications to complex analysis in several variables.
\end{abstract}

\section{Introduction}

In this paper we present various generalized versions of the Frobenius theorem on involutivity in explicit forms. Let $M$ be a smooth $\left(C^{\infty}\right)$ manifold of dimension $m$ and $\theta=\left(\theta^{1}, \ldots, \theta^{s}\right)$ be a system of smooth 1 -forms that are linearly independent at every point of $M$. Then the Frobenius theorem states that if $\theta$ satisfies the integrability condition (1.6), then locally there is a $s$ parameter family of integral manifolds of maximal dimension $p:=m-s$. This theorem can be generalized in two directions:

One is deciding whether there exists a $s^{\prime}\left(s^{\prime}<s\right)$ parameter family of integral manifolds, which we shall discuss in $\S 2$. Generalization in this direction is essentially reducing the Pfaffian system $\theta$ to the submanifold where torsions vanish. It turned out that this is precisely the reduction process (Step 1 of $\S 1$ ) in prolongation of a Pfaffian system to an equivalent involutive system. The other is finding integral manifolds of lower dimensions (less than $p$ ), which we shall discuss in $\S 3$. This is basically linear algebra of computing the rank of the torsion tensor $d \theta$ modulo $\theta$. In the case $s=1$, the classical theorem of Darboux (Theorem 3.10) asserts that if the rank of the system is $r$, then $M$ is foliated by integral manifolds of dimension $m-(r+1)$. The case $r=0$ is the Frobenius integrability condition (1.6) and $M$ is foliated by integral manifolds

Received December 21, 2007.

2000 Mathematics Subject Classification. 35N10, 58A15, 32F25.

Key words and phrases. Pfaffian system, involutivity, integral manifold, foliation.

The author was partially supported by KRF 2005-070-C00007 of Korea Research Foundation. 
of dimension $m-1$. Hence, Darboux theorem is a generalization of the second kind of the special case $s=1$ of the Frobenius theorem. Generalized Frobenius theorems are useful in finding submanifolds with required properties as we see in [17] and [15]. Also, generalized Frobenius theorems are very efficiently used in finding all the compatibility conditions for a given overdetermined PDE system of generic type in order to find general solutions, for which the readers are referred to the author's survey articles [13] and [14]. Existence of a single (isolated) integral manifolds of maximal dimension $p$ belongs to both the first kind and the second kind of generalization, which appears explicitly in [2] and [24]. The author independently obtained the results together with further generalizations that we presented in this paper. Afterwards, he finds some of the ideas are already in E. Cartan, R. Bryant, or even in the writings of Pfaff and Darboux. The purpose of this paper is to write down the various versions of the generalized Frobenius theorem in explicit form, which is hard to find in literature, in order to use as a basic reference in the future. The advantage of the Pfaffian system of Frobenius type (definition is given in $\S 1$ ) is that the notion of involutivity is the same as the integrability condition (1.6) and the existence theory works in $C^{\infty}$ category, or in $C^{k}$-category for sufficiently large $k$, while in the classical theory of E. Cartan on exterior differential system the existence of solutions is proved by using the Cauchy-Kowalewski theorem, assuming the analyticity $\left(C^{\omega}\right)$ of the data.

Real valued functions $\rho_{1}, \ldots, \rho_{d}$ defined on $M$ shall be said to be nondegenerate if $d \rho_{1} \wedge \cdots \wedge d \rho_{d} \neq 0$ modulo $\rho_{1}, \ldots, \rho_{d}$. Our argument in this paper is purely local: we work on a neighborhood of a reference point and often we shrink this neighborhood to a smaller one as our argument proceeds.

\section{$\S 1$. History and some background}

For a smooth $\left(C^{\infty}\right)$ vector field $X=\sum_{i=1}^{m} a^{i}(x) \partial / \partial x_{i}$ defined on an open subset $U \subset \mathbb{R}^{m}$ a constant of motion, or a first integral, is a function that remains constant along each integral curve of $X$. There are $n-1$ functionally independent constants of motion that can be obtained by solving the total differential equations

$$
\frac{d x_{1}}{a^{1}(x)}=\frac{d x_{2}}{a^{2}(x)}=\cdots=\frac{d x_{m}}{a^{m}(x)} .
$$

When multiple vector fields

$$
X_{j}=\sum_{i=1}^{m} a_{j}^{i}(x) \partial / \partial x_{i}, \quad j=1, \ldots, p
$$

are given F. Deahna [9] proved that if $X_{j}$ 's satisfy the integrability conditions (1.1), then there exist $s:=m-p$ functions whose common level sets are the integral manifolds. The problem was finding independent solutions of the 
overdetermined PDE system of homogeneous linear equations of first order:

$$
\sum_{i=1}^{m} a_{j}^{i}(x) \frac{\partial u}{\partial x_{i}}=0 \quad j=1, \ldots, p .
$$

Theorem 1.1, which we call the Frobenius theorem, was first proved by A. Clebsch [7]. G. Frobenius is responsible for applying the theorem to Pfaffian system (Theorem 1.2), thus paving the way for its usage in differential topology (cf. Wikipedia, Frobenius theorem). Also, it was Frobenius who first used $d$; the exterior derivative applied to Pfaffian systems, and called it bilinear covariant (cf. [1]). Expressing the theorem in terms of differential forms and their exterior derivative $d$ has great advantages in computation.

Now we recall the Frobenius theorem as in (cf. [25, Chapter 1]). Let $M^{m}$ be a smooth manifold of dimension $m$. Let $X_{1}, \ldots, X_{p}$ be smooth vector fields that are linearly independent at every point. Let $\mathcal{D}$ be the distribution of $p$-dimensional tangent planes spanned by $X_{1}, \ldots, X_{p}$. A submanifold $N$ of dimension $p^{\prime} \leq p$ is called an integral manifold of $\mathcal{D}$ if at every point $x \in N$, $T_{x} N \subset \mathcal{D}$. The distribution $\mathcal{D}$ is said to be integrable if

$$
\left[X_{i}, X_{j}\right](x) \in \mathcal{D}, \quad \forall x \in M .
$$

The Frobenius theorem states:

Theorem 1.1. Suppose that $\mathcal{D}$ is a smooth distribution spanned by a set of smooth vector fields $X_{1}, \ldots, X_{p}$ that satisfies the integrability condition (1.1). Then at any point $x \in M$ there exists a unique smooth integral manifold $N$ of maximal dimension $p$ through $x$.

Let

$$
\theta=\left(\theta^{1}, \ldots, \theta^{s}\right), \quad s+p=m
$$

be a system of linearly independent 1-forms that defines $\mathcal{D}$, that is, for $(x, V)$ a tangent vector, $V \in \mathcal{D}$ if and only if $\theta^{\alpha}(V)=0$ for each $\alpha=1, \ldots, s$. Typically, $\theta$ is found by taking smooth vector fields $Y_{1}, \ldots, Y_{s}, p+s=m$, so that

$$
X_{1}, \ldots, X_{p}, Y_{1}, \ldots, Y_{s}
$$

span the whole tangent space at every point of $M$ and then taking the dual 1 -forms

$$
\omega^{1}, \ldots, \omega^{p}, \theta^{1}, \ldots, \theta^{s} .
$$

Consider the exterior algebra of differential forms $\Omega:=\bigoplus_{k=0}^{m} \Omega^{k}$, where $\Omega^{k}$ is the set of smooth $k$-forms and $\Omega^{0}:=C^{\infty}(M)$ is the ring of smooth functions on $M$. Each $\Omega^{k}$ is a module over $C^{\infty}(M)$. A subalgebra $\mathcal{I}$ is called an algebraic ideal if $\mathcal{I} \wedge \Omega \subset \mathcal{I}$ and if the following additional condition is satisfied: if $\phi=\sum_{k=0}^{m} \phi_{k} \in \mathcal{I}$, where $\phi_{k} \in \Omega^{k}$, then each component $\phi_{k} \in \mathcal{I}$ (homogeneity condition). 
Now let $\mathcal{I}$ be the algebraic ideal generated by $\theta$, which is the set of all elements of $\Omega$ of the form $\sum_{\alpha=1}^{s} \theta^{\alpha} \wedge \phi^{\alpha}, \phi^{\alpha} \in \Omega^{k}$ for some $k$. The ideal $\mathcal{I}$ is said to be closed if

$$
d \mathcal{I} \subset \mathcal{I}
$$

Then the following are equivalent:

a) $\mathcal{D}$ is integrable in the sense that its generating vector fields satisfy (1.1);

b) $\mathcal{I}$ is closed;

c) For each $\alpha=1, \ldots, s$,

$$
d \theta^{\alpha}=0 \bmod \left(\theta^{1}, \ldots, \theta^{s}\right),
$$

and we may state the Frobenius theorem as follows:

Theorem 1.2. Let $M$ be a smooth manifold of dimension $m$ and let $\theta=$ $\left(\theta^{1}, \ldots, \theta^{s}\right)$ be a system of smooth 1-forms that are linearly independent at every point of $M$. If $\theta$ satisfies the integrability condition (1.6), then for any point $x \in M$ there exists a unique integral manifold $N$ of dimension $p:=m-s$ through $x$. Therefore, $M$ is foliated by s-parameter family of integral manifolds.

The Frobenius' setting of Pfaffian system with $d$ was studied further by G. Darboux [8] and later developed to be the theory exterior differential system by E. Cartan $[3,4]$. In Cartan's theory the basic notions are prolongation and involutivity, which we now briefly review. We refer the readers to our standard references [1], [11], and [18].

Let $M$ be a smooth $\left(C^{\infty}\right)$ manifold of dimension $m$ and let $\theta^{1}, \ldots, \theta^{s}$, $\omega^{1}, \ldots, \omega^{p}, s+p \leq m$, be a set of linearly independent smooth 1-forms on $M$. We are concerned with the problem of finding a smooth submanifold $N \subset M$ of dimension $p$ which satisfies

$$
\begin{aligned}
& \left.\theta^{\alpha}\right|_{N}=0, \quad \alpha=1, \ldots, s \quad \text { (Pfaffian system) } \\
& \left.\Omega\right|_{N} \neq 0, \quad \text { where } \Omega=\omega^{1} \wedge \cdots \wedge \omega^{p} \quad \text { (independence condition). }
\end{aligned}
$$

Such a submanifold $N$ is called an integral manifold of dimension $p$ satisfying the independence condition, or simply a 'solution' of (1.7). To find a solution of (1.7) we consider subbundles $I \subset J \subset T^{*} M$. Here $I=\left\langle\theta^{1}, \ldots, \theta^{s}\right\rangle$ and $J=\left\langle\theta^{1}, \ldots, \theta^{s}, \omega^{1}, \ldots, \omega^{p}\right\rangle$, where $\langle\cdots\rangle$ denotes the linear span of what are inside. Let $\mathcal{D}$ be the $(m-s)$-dimensional plane field annihilated by $\theta^{1}, \ldots, \theta^{s}$. For $k=1, \ldots, p$, an integral manifold of (1.7) of dimension $k$ is a submanifold of $M$ of dimension $k$ whose tangent spaces are contained in $\mathcal{D}$. An integral manifold $N$ of dimension $p$ such that $\left.\Omega\right|_{N} \neq 0$ is a solution of (1.7). If $N$ is an integral manifold of (1.7), then $\left.\theta^{\alpha}\right|_{N}=0$, and therefore, $\left.d \theta^{\alpha}\right|_{N}=0$ for each $\alpha=1, \ldots, s$. A $k$-dimensional integral element is a $k$-dimensional subspace $(x, E)$ of $T_{x} M$, for some $x \in M$, on which $\theta^{\alpha}=0$ and $d \theta^{\alpha}=0$ for all $\alpha=1, \ldots, s$. By $V(I, J)$ we denote the set of all $p$-dimensional integral elements $(x, E)$ satisfying $\left.\Omega\right|_{E} \neq 0$. Basic idea of the theory is that we can find 
a solution by constructing $k$-dimensional integral manifold $N^{k}$ with $N^{k-1}$ as initial data, inductively for $k=1, \ldots, p$, so that we have a nested sequence of integral manifolds

$$
N^{0} \subset N^{1} \subset \cdots \subset N^{p}
$$

Let

$$
\{x\}=E^{0} \subset E^{1} \subset \cdots \subset E^{p}=E
$$

be the corresponding flag of integral elements. The notion of involutivity is the existence of such a flag for each element of $V(I, J)$ so that the Cauchy problem is well-posed in each step and the solutions to the $(k+1)^{s t}$ Cauchy problem remain solutions to the family of $k^{\text {th }}$ Cauchy problem with data smoothly changing in $(k+1)^{s t}$ direction. If the system is analytic $\left(C^{\omega}\right)$ one can construct such a nested sequence of integral manifolds by using the Cauchy-Kowalewski theorem. This is the idea of the Cartan-Kähler theorem which asserts that an involutive analytic Pfaffian system has analytic solutions. If $(I, J)$ is not involutive we construct an involutive system which is equivalent to the original system by repeating the process of the following two steps:

Step 1. Reduce (1.7) to a submanifold $M^{\prime} \subset M$ so that $V^{\prime}(I, J) \rightarrow M^{\prime}$ is surjective.

Let $M_{1}$ be the image of $V(I, J) \rightarrow M$. If $M=M_{1}$, then we do nothing. If $M_{1} \neq M$, then we note that any integral manifold of $(I, J)$ must lie in $M_{1}$, and so we set

$$
V_{1}(I, J)=\left\{(x, E) \in V(I, J): E \subset T_{x} M_{1}\right\} .
$$

Now consider the projection

$$
V_{1}(I, J) \rightarrow M_{1}
$$

with image $M_{2}$. If $M_{2}=M_{1}$ we stop; otherwise we continue as before. Eventually we arrive either at the empty set, in which case $(I, J)$ has no integral manifolds, or else at $M^{\prime}$ with $V^{\prime}(I, J) \rightarrow M^{\prime}$ being surjective and with all $(x, E) \in V^{\prime}(I, J)$ satisfying $E \subset T_{x} M^{\prime}$.

Step 2. Assuming $V(I, J) \rightarrow M$ is surjective we do prolongation.

To recall the definitions, let $G_{p}(M)$ be the Grassmann bundle of $p$-planes in $T M$. Let $\pi^{1}, \ldots, \pi^{r}$ be a set of 1 -forms so that

$$
\theta^{1}, \ldots, \theta^{s}, \omega^{1}, \ldots, \omega^{p}, \pi^{1}, \ldots, \pi^{r}
$$

form a basis of $T^{*} M$. Let $(x, E) \in V(I, J)$. Since $\left.\Omega\right|_{E} \neq 0$, on a neighborhood of $(x, E) \in G_{p}(M)$ we have $\theta^{\alpha}=m_{\rho}^{\alpha} \omega^{\rho}, \pi^{\epsilon}=\ell_{\rho}^{\epsilon} \omega^{\rho}$, (summation convention for $\rho=1, \ldots, p)$ and $\Omega \neq 0$. Thus $\left\{m_{\rho}^{\alpha}, \ell_{\rho}^{\epsilon}\right\}$ are local fibre coordinates in $G_{p}(M)$. The canonical system on $G_{p}(M)$ is the set of the tautological 1-forms

$$
\begin{aligned}
& \theta^{\alpha}-m_{\rho}^{\alpha} \omega^{\rho}, \quad \alpha=1, \ldots, s \\
& \pi^{\epsilon}-\ell_{\rho}^{\epsilon} \omega^{\rho}, \quad \epsilon=1, \ldots, r,
\end{aligned}
$$


where the summation convention is used for $\rho=1, \ldots, p$. The first prolongation $\left(I^{(1)}, J^{(1)}\right)$ is the restriction to $M^{(1)}:=V(I, J) \subset G_{p}(M)$ of the canonical system. Since $m_{\rho}^{\alpha}=0$ on $V(I, J)$ the problem of finding a solution of (1.7) is equivalent to finding a submanifold $N^{(1)} \subset M^{(1)}$ of dimension $p$ satisfying

$$
\begin{aligned}
& \left.\theta^{\alpha}\right|_{N^{(1)}}=0,\left.\quad\left(\pi^{\epsilon}-\ell_{\rho}^{\epsilon} \omega^{\rho}\right)\right|_{N^{(1)}}=0, \\
& \left.\Omega\right|_{N^{(1)}} \neq 0 .
\end{aligned}
$$

Integral manifolds of $(I, J)$ and those of $\left(I^{(1)}, J^{(1)}\right)$ are in a one-to-one correspondence. The $k$-th prolongation $\left(I^{(k)}, J^{(k)}\right)$ on $M^{(k)}=V\left(I^{(k-1)}, J^{(k-1)}\right)$ is defined inductively to be the first prolongation of $\left(I^{(k-1)}, J^{(k-1)}\right)$ on $M^{(k-1)}$. We have a version of the Cartan-Kuranishi theorem [18]:

Theorem 1.3. Let $\left(I^{(k)}, J^{(k)}\right), k=1,2, \ldots$, be the sequence of prolongations of $(I, J)$. Suppose that, for each $k, M^{(k)}$ is a smooth submanifold of $G_{p}\left(M^{(k-1)}\right)$ and that the projection $M^{(k)} \rightarrow M^{(k-1)}$ is a surjective submersion. Then there is $k_{0}$ such that prolongations $\left(I^{(k)}, J^{(k)}\right)$ are involutive for $k \geq k_{0}$.

Now we discuss the Pfaffian system of Frobenius type. Consider the algebraic ideals $\mathcal{I}$ and $\mathcal{J}$ generated by $\left\{\theta^{1}, \ldots, \theta^{s}\right\}$ and $\left\{\theta^{1}, \ldots, \theta^{s}, \omega^{1}, \ldots, \omega^{p}\right\}$, respectively. (1.7) is quasi-linear if $d \mathcal{I} \subset \mathcal{J}$, namely,

$$
d \theta^{\alpha}=\sum_{\beta=1}^{s} \phi_{\beta}^{\alpha} \wedge \theta^{\beta}+\sum_{\rho=1}^{p} \psi_{\rho}^{\alpha} \wedge \omega^{\rho}
$$

for some 1-forms $\phi_{\beta}^{\alpha}, \psi_{\rho}^{\alpha}$, for each $\alpha=1, \ldots, s$. Existence of solutions has been studied mainly for the quasi-linear systems. (1.7) is said to be of Frobenius type if $s+p=m$, that is, if $\left\{\theta^{1}, \ldots, \theta^{s}, \omega^{1}, \ldots, \omega^{p}\right\}$ is a coframe of $M$. It is easy to see that Frobenius types are quasi-linear. In this case no further equations are obtained by prolongation and the existence of general integral manifolds is determined only by Step 1 of $\S 2$. The notion of involutivity is very subtle as we see in [1]. However, for $V(I, J)$ of Frobenius type the following are equivalent (see [11, Chapter 3]):

i) $V(I, J) \rightarrow M$ is surjective.

ii) $(I, J)$ is integrable in the sense of the Frobenius theorem.

iii) $(I, J)$ is invoultive.

The author studies in [14] the reduction to a submanifold of a Pfaffian system of Frobenius type based on the following lemma, whose proof is easy.

Lemma 1.4. Let $M$ be a smooth manifold of dimension $m$. Let $\theta:=\left(\theta^{1}, \ldots\right.$, $\left.\theta^{s}\right)$ be a set of independent 1-forms on $M$ and $\mathcal{D}:=\langle\theta\rangle^{\perp}$ be the $(m-s)$ dimensional plane field annihilated by $\theta$. Suppose that $N$ is a submanifold of $M$ of dimension $n:=m-r$ for some $r \leq s$, defined by $T_{1}=\cdots=T_{r}=0$, where $T_{i}$ are smooth real-valued functions of $M$ such that $d T_{1} \wedge \cdots \wedge d T_{r} \neq 0$. Then the following are equivalent:

(i) $\mathcal{D}$ is tangent to $N$. 
(ii) $d T_{j} \equiv 0 \bmod \theta$, on $N$ for each $j=1, \ldots, r$.

In (ii) $\bmod \theta$ means that modulo the algebraic ideal $\mathcal{I}$. Thus (ii) is equivalent to saying that for each $j=1, \ldots, r$ we have $d T_{j} \wedge \theta^{1} \wedge \cdots \wedge \theta^{s}=0$ on $N$. Our basic observation is the following algorithm for Step 1: For each $\alpha=1, \ldots, s$, set

$$
d \theta^{\alpha}=T_{i j}^{\alpha} \omega^{i} \wedge \omega^{j} \quad \bmod \theta, \quad(\text { summation convention for } i, j=1, \ldots, p)
$$

where $T_{i j}^{\alpha}$ are skew symmetric in $(i j)$. Let $\mathcal{T}_{1}$ be the set of functions $\left\{T_{i j}^{\alpha}\right\}$. If $\mathcal{T}_{1}$ are identically zero, then $V(I, J) \rightarrow M$ is surjective, which is the Frobenius integrability condition for $\theta$, and by Frobenius theorem we have $(m-p)$-parameter family of integral manifolds. Otherwise, let $M_{1}$ be the common zero set of $\mathcal{T}_{1}$ and set

$$
d T_{i j}^{\alpha}=T_{i j, k}^{\alpha} \omega^{k} \bmod \theta
$$

Let $\mathcal{T}_{2}$ be the set of functions $T_{i j, k}^{\alpha}$. If $\mathcal{T}_{2}$ are identically zero on $M_{1}$, then $V_{1}(I, J) \rightarrow M_{1}$ is surjective, and by Frobenius theorem there exist $\left(\operatorname{dim} M_{1}-p\right)$ parameter family of solutions. If $\mathcal{T}_{2}$ are not identically zero, let $M_{2}$ be the submanifold of $M_{1}$ defined by $\mathcal{T}_{2}=0$ and continue as before. Eventually we arrive either at an empty set, in which case there is no integral manifolds, or at an integrable Pfaffian system on a submanifold $M^{\prime} \subset M$, in which case there exist $\left(\operatorname{dim} M^{\prime}-p\right)$-parameter family of integral manifolds.

\section{$\S 2 . s^{\prime}$ parameter family of integral manifolds of maximal dimension}

Based on Lemma 1.4 and the remarks thereafter, we discuss in this section the existence of $s^{\prime}\left(s^{\prime}<s\right)$ parameter family of maximal dimensional integral manifolds. In particular, the case $s^{\prime}=0$ is the existence of a single isolated integral manifold of dimension $p$. Let $M^{m}$ and $\theta=\left(\theta^{1}, \ldots, \theta^{s}\right)$ be the same as in the Frobenius theorem (Theorem 1.2), and let $\omega^{1}, \ldots, \omega^{p}, p+s=m$, be a complementary set of 1-forms.

In order to reduce the Pfaffian system $\theta=0$ to a submanifold we want, by Lemma 1.4, to find real valued functions $\rho_{1}, \ldots, \rho_{d}$ such that

i) $d \rho_{1} \wedge \cdots \wedge d \rho_{d} \neq 0$,

ii) $d \rho_{j} \equiv 0 \bmod \left(\rho_{1}, \ldots, \rho_{d}, \theta^{1}, \ldots, \theta^{s}\right)$ for $j=1, \ldots, d$.

Then the problem is reduced to $M^{\prime}:=\left\{x \in M: \rho_{j}(x)=0, j=1, \ldots, d\right\}$. Let $m^{\prime}=m-d$ be the dimension of $M^{\prime}$. Let $i: M^{\prime} \hookrightarrow M$ be the inclusion map. Then ii) implies that the rank of $i^{*} \theta$ is constantly $s^{\prime}=s-d$. If the torsions vanish on $M^{\prime}$, then by the Frobenius theorem there exists a $s^{\prime}$-parameter family of integral manifolds of dimension

$$
m^{\prime}-s^{\prime}=(m-d)-(s-d)=m-s=p .
$$

In practice, one can reduce to a submanifold of codimension $d=1$ by finding $\rho$ : we have 
Theorem 2.1. Let $M$ be a $C^{\infty}$ manifold of dimension $m$ and $\theta=\left(\theta^{1}, \ldots, \theta^{s}\right)$ be a system of $C^{\infty} 1$-forms that are linearly independent at every point of $M$. Suppose that a $C^{\infty}$ real valued function $\rho$ on $M$ is non-degenerate and satisfies

$$
d \rho \equiv 0 \bmod (\rho, \theta) .
$$

Then

$$
\theta^{1} \wedge \cdots \wedge \theta^{s} \equiv 0 \quad \bmod (\rho, d \rho)
$$

Proof. The hypothesis implies that

$$
d \rho=\rho \psi+\sum_{i=1}^{s} a_{i} \theta^{i}
$$

for some $C^{\infty}$ functions $a_{i}$ and a 1-form $\psi$. Let $(x, V)$ be an arbitrary tangent vector at $x$ to the zero set of $\rho$. Since $d \rho(x) \neq 0$, some of $a_{i}$ are non-zero at $x$. Evaluating $(2.1)$ at $(x, V)$ we have

$$
\sum_{i=1}^{s} a_{i}(x) \theta^{i}(V)=0 .
$$

This implies that

$$
i^{*}\left(\theta^{1} \wedge \cdots \wedge \theta^{s}\right)=0
$$

where

$$
i:\{x \in M: \rho(x)=0\} \hookrightarrow M
$$

is the inclusion map, which is equivalent to the conclusion.

Example 2.2 (Reduction to a system without solutions on a submanifold). In $\mathbb{R}^{4}=\{(x, y, z, w)\}$ consider the following two independent 1-forms

$$
\theta=\left(\theta^{1}, \theta^{2}\right), \quad \text { where } \theta^{1}=d z+x d y, \quad \theta^{2}=d w+w d x .
$$

Let $\rho(x, y, z, w)=w$ and let $M^{\prime}=\{w=0\}$. Since $d \rho=d w \neq 0$ and $d \rho=d w \equiv$ $0 \bmod \left(w, d w, \theta^{1}, \theta^{2}\right)$, the Pfaffian system $\theta=0$ reduces to $M^{\prime}$.

But there is no integral manifolds in $i: M^{\prime} \hookrightarrow \mathbb{R}^{4}$ for the following reason: $i^{*} \theta^{2}=0, i^{*} \theta^{1}=d z+x d y$ and

$$
d\left(i^{*} \theta^{1}\right)=i^{*}\left(d \theta^{1}\right)=d x \wedge d y \neq 0 \quad \bmod \left(i^{*} \theta\right) .
$$

Example 2.3 (Reduction to an involutive system on a submanifold). In $\mathbb{R}^{4}=$ $\{(x, y, z, w)\}$ given 1 -forms

$$
\theta=\left(\theta^{1}, \theta^{2}\right), \quad \text { where } \theta^{1}=d z+z d y, \quad \theta^{2}=d w+w(1+y) d x .
$$

As in Example 2.2 we can easily check that the Pfaffian system $\left(\mathbb{R}^{4} ; \theta^{1}, \theta^{2}\right)$ reduces the submanifold $M^{\prime}=\{w=0\}$. In the submanifold $i: M^{\prime} \hookrightarrow \mathbb{R}^{4}$ we have $i^{*} \theta^{2}=0, i^{*} \theta^{1}=d z+z d y$ and

$$
\begin{aligned}
i^{*}\left(d \theta^{1}\right) & =d z \wedge d y \\
& =-z d y \wedge d y \bmod \theta \\
& =0
\end{aligned}
$$


Hence, the reduced system $\left(M^{\prime}, \theta^{1}\right)$ is involutive so that there exists a 1parameter family of integral manifolds of dimension 2. Similarly, $M^{\prime \prime}=\{z=0\}$ gives another reduction. Then for the inclusion map $i: M^{\prime \prime} \hookrightarrow \mathbb{R}^{4}$, we have

$$
d\left(i^{*} \theta^{2}\right)=i^{*}\left(d \theta^{2}\right)=w d y \wedge d x \neq 0 .
$$

Therefore, torsion is $w$. In fact, the plane $w=0, z=0$ is the only integral manifold that is contained in $M^{\prime \prime}$.

Example 2.4 (Reduction to a pair of involutive systems on submanifolds). In $\mathbb{R}^{4}=\{(x, y, z, w)\}$ we consider 1-forms $\theta=\left(\theta^{1}, \theta^{2}\right)$, where

$$
\theta^{1}=d z+w f(x, y) d w, \quad \theta^{2}=d w+z g(x, y) d z .
$$

As in Example 2.2 we can easily check that the Pfaffian system $\left(\mathbb{R}^{4} ; \theta^{1}, \theta^{2}\right)$ reduces to the submanifold $M^{\prime}=\{w=0\}$. In the submanifold $i: M^{\prime} \hookrightarrow \mathbb{R}^{4}$ we have $i^{*} \theta^{1}=d z, i^{*} \theta^{2}=z g(x, y) d z$, so that the original system reduces to $\left(M^{\prime}, \theta^{1}\right)$. Since $d\left(i^{*} \theta^{1}\right)=0$, the reduced system is involutive. Similarly, $M^{\prime \prime}=$ $\{z=0\}$ gives another reduction. Then for the inclusion map $i: M^{\prime \prime} \hookrightarrow \mathbb{R}^{4}$, we have $i^{*} \theta^{1}=w f(x, y) d w, i^{*} \theta^{2}=d w$, so that the original system reduces to $\left(M^{\prime \prime}, \theta^{2}\right)$. Since $d\left(i^{*} \theta^{2}\right)=0$, the reduced system $\left(M^{\prime \prime}, \theta^{2}\right)$ is involutive.

Next we discuss the cases where there exists exactly one integral manifold of dimension $p$. These cases may be regarded as reduction of the Pfaffian system to $p$-dimensional submanifold. For possible applications we discuss the cases with degenerate torsion, that is, torsion $T$ with $d T(0)=0$ at the reference point:

On $\mathbb{R}^{3}=\{(x, y, z)\}$ consider a 1 -form

$$
\theta=d z+f(x, y, z) d y
$$

where $f(x, y, z)$ is a smooth $\left(C^{\infty}\right)$ real valued function defined on an open neighborhood of the origin. We have

$$
\begin{aligned}
d \theta & =\left(f_{x} d x+f_{y} d y+f_{z} d z\right) \wedge d y \\
& =f_{x} d x \wedge d y \bmod \theta
\end{aligned}
$$

Therefore, the torsion is

$$
T=f_{x} .
$$

If $T$ is identically zero, then by the Frobenius theorem there exists a 1-parameter family of integral manifolds.

In order construct examples with singular torsion sets we set

$$
T=f_{x}=z(z-g(x, y))=z^{2}-z g(x, y) .
$$

I want $z=0$ is the only integral manifold, so that we require

$$
\left\{\begin{array}{l}
f(x, y, 0)=0 \\
f_{x}=z^{2}-z g(x, y) .
\end{array}\right.
$$


Second condition implies that

$$
f(x, y, z)=z^{2} x-z G(x, y),
$$

where $G_{x}=g$. Now any pair $(G, g)$ with $G_{x}=g$ yields the torsion $(2.2)$.

Example 2.5 (Degenerate torsion with an isolated integral manifold). $G(x, y z)=x^{2}, \quad g(x, y)=2 x$ : Let $\theta=d z+\left(z^{2} x-z x^{2}\right) d y$. Then $d \theta \equiv$ $\left(z^{2}-2 z x\right) d x \wedge d y \bmod \theta$. Therefore, $T=z(z-2 x)$. The zero set of $T$ is two planes intersecting along $y$-axis, among which the plane $z=0$ is an integral manifold.

Example 2.6 (Degenerate torsion with an isolated integral manifold). Let $f_{x}=z\left(z^{2}-x^{2}-y^{2}\right)$, so that $f(x, y, z)=z^{3} x-z x^{3} / 3-z y^{2} x$. Then the zero set of the torsion is given by $z\left(z^{2}-x^{2}-y^{2}\right)=0$. This variety is the union of the plane $z=0$ and the cone $z^{2}-x^{2}-y^{2}=0 . z=0$ is an integral manifold.

\section{$\S 3$. Integral manifolds of lower dimensions}

First of all, we prove the following:

Theorem 3.1. Let $M^{m}$ be a smooth manifold and let $\theta:=\left(\theta^{1}, \ldots, \theta^{s}\right)$ be a system of smooth 1-forms that are linearly independent at every point of $M$. Let $n$ be an integer such that $2 \leq n \leq p:=m-s$. Suppose that $i: N^{n} \hookrightarrow M^{m}$ is a submanifold of dimension $n$, defined by $\rho_{1}=\cdots=\rho_{m-n}=0$, where $\rho_{j}$ are smooth real-valued functions of $M$ such that $d \rho_{1} \wedge \cdots \wedge d \rho_{m-n} \neq 0$. Then the following are equivalent:

(i) $i^{*} \theta^{\alpha}=0, \quad \alpha=1, \ldots, s$;

(ii) $\forall \alpha=1, \ldots, s, \theta^{\alpha} \equiv 0 \bmod \left(\rho_{1}, \ldots, \rho_{m-n}, d \rho_{1}, \ldots, d \rho_{m-n}\right)$.

Lemma 3.2. Let $(t, x)$, where $t=\left(t_{1}, \ldots, t_{d}\right), x=\left(x_{1}, \ldots, x_{n}\right)$, be the standard coordinates of $\mathbb{R}^{d+n}$. Suppose that $f$ is a $C^{\infty}$ function defined on a neighborhood of the origin such that $f(0, x)=0$. Then $f(t, x)=\sum_{j=1}^{d} t_{j} g^{j}(t, x)$ for some $C^{\infty}$ functions $g^{1}, \ldots, g^{d}$ defined on a smaller neighborhood of the origin.

Proof.

$$
\begin{aligned}
f(t, x) & =\int_{0}^{1} \frac{\partial}{\partial \tau} f(\tau t, x) d \tau \\
& =\int_{0}^{1} \sum_{j=0}^{d} t_{j} f_{j}(\tau t, x) d \tau, \quad \text { where } f_{j}=\frac{\partial f}{\partial t_{j}} \\
& =\sum_{j=1}^{d} t_{j} \int_{0}^{1} f_{j}(\tau t, x) d \tau .
\end{aligned}
$$

Let $g^{j}(t, x)=\int_{0}^{1} f_{j}(\tau t, x) d \tau$ for each $j=1, \ldots, d$. Then it is standard to show that $g^{j}$ are $C^{\infty}$. 
Proof of Theorem 3.1. (i) $\Rightarrow$ (ii): Choose independent 1 -forms $\omega^{1}, \ldots, \omega^{n}$ so that

$$
d \rho_{1}, \ldots, d \rho_{m-n}, \omega^{1}, \ldots, \omega^{n}
$$

$\operatorname{span} T^{*} M$. Then

$$
i^{*}\left(\omega^{1} \wedge \cdots \wedge \omega^{n}\right) \neq 0
$$

Set

$$
\theta^{\alpha}=\sum_{j=1}^{m-n} a^{\alpha j} d \rho_{j}+\sum_{j=1}^{n} b_{j}^{\alpha} \omega^{j} .
$$

Since $i^{*} \theta^{\alpha}=0$ and $i^{*}\left(d \rho_{j}\right)=0$, pulling back (3.1) by $i$ we have

$$
0=\sum_{j=1}^{n} b_{j}^{\alpha}\left(i^{*} \omega^{j}\right)
$$

Therefore, for each $\alpha, j$, we have $b_{j}^{\alpha}=0$ on $N$, which implies by Lemma 3.2

$$
b_{j}^{\alpha}=\sum_{k=1}^{m-n} h_{j}^{\alpha k} \rho_{k}
$$

for some smooth function $h_{j}^{\alpha k}$. Substituting (3.2) for $b_{j}^{\alpha}$ in (3.1) we have

$$
\theta^{\alpha}=\sum_{j=1}^{m-n} a^{\alpha j} d \rho_{j}+\sum_{j=1}^{n} \sum_{k=1}^{m-n} \rho_{k} h_{j}^{\alpha k} \omega^{j} .
$$

(ii) $\Rightarrow$ (i) : Suppose that

$$
\theta^{\alpha}=\sum_{j=1}^{m-n} \rho_{j} \psi^{\alpha j}+\sum_{j=1}^{m-n} h^{\alpha j} d \rho_{j}
$$

for some 1-forms $\psi^{\alpha j}$ and smooth functions $h^{\alpha j}$. Apply any tangent vector $(x, V) \in T N$ to (3.4). Since $\rho_{j}(x)=0$ and $d \rho_{j}(V)=0$, we have $\theta^{\alpha}(V)=0$, which implies that $i^{*} \theta^{\alpha}=0$.

Now we study by using Theorem 3.1, the existence of integral manifold $i: N^{n} \hookrightarrow M^{m}, 2 \leq n \leq p$, of the Pfaffian system

$$
\theta^{\alpha}=0, \quad \alpha=1, \ldots, s, \quad s+p=m .
$$

Suppose that $N$ is an integral manifold of (3.5). Then $i^{*} \theta^{\alpha}=0$ implies that $d\left(i^{*} \theta^{\alpha}\right)=i^{*}\left(d \theta^{\alpha}\right)=0$. Let $\omega^{1}, \ldots, \omega^{p}$ be the complementary set of 1 -forms. We set as usual

$$
d \theta^{\alpha}=\sum_{i, j=1}^{p} T_{i j}^{\alpha} \omega^{i} \wedge \omega^{j} \quad \bmod \theta, \quad \alpha=1, \ldots s,
$$


where $T_{j i}^{\alpha}=-T_{i j}^{\alpha}$. Consider $\left(\begin{array}{l}p \\ 2\end{array}\right):=p(p-1) / 2$ linearly independent differential 2-forms $\omega^{i} \wedge \omega^{j}$ arranged in lexico-graphical order. Let

$$
\mathcal{T}=\left(T_{i j}^{\alpha}\right)
$$

be the matrix of size $s \times\left(\begin{array}{l}p \\ 2\end{array}\right)$. We shall call $\mathcal{T}$ torsion of the Pfaffian system $(3.5)$.

Proposition 3.3. Let $M$ be a smooth manifold of dimension $m$ and let $\theta^{1}, \ldots$, $\theta^{s}, \omega^{1}, \ldots, \omega^{p}$ be a system of smooth 1 -forms as in (3.5)-(3.6). Suppose that $N$ is an integral manifold of (3.5) of dimension $n, 2 \leq n \leq p$. Then there exists $\left(\begin{array}{l}p \\ 2\end{array}\right) \times\left(\begin{array}{l}n \\ 2\end{array}\right)$ matrix valued smooth function $A$ of rank $\left(\begin{array}{l}n \\ 2\end{array}\right)$ defined on $N$ such that

$$
\mathcal{T} A=0
$$

In particular, if $N^{p}$ is an integral manifold of maximal dimension, then $\mathcal{T}=0$ on $N^{p}$.

Proof. After re-ordering if necessary, we may assume that $\left.\omega^{1} \wedge \cdots \wedge \omega^{n}\right|_{N} \neq 0$. Set

$$
\left.\omega^{\lambda}\right|_{N}=\left.\sum_{i=1}^{n} a_{i}^{\lambda} \omega^{i}\right|_{N}, \quad \lambda=n+1, \ldots, p .
$$

Then the restriction to $N$ of (3.6) reads

$$
\begin{aligned}
0 & =\sum_{\substack{i<j \\
i, j=1, \ldots, n}} \tau_{i j}^{\alpha} \omega^{i} \wedge \omega^{j}, \text { where } \\
\tau_{i j}^{\alpha} & =T_{i j}^{\alpha}+\sum_{\mu=n+1}^{p} T_{i \mu}^{\alpha} a_{j}^{\mu}-\sum_{\lambda=n+1}^{p} T_{j \lambda}^{\alpha} a_{i}^{\lambda}+\sum_{\substack{\lambda<\mu \\
\lambda, \mu=n+1, \ldots, p}} T_{\lambda \mu}^{\alpha}\left(a_{i}^{\lambda} a_{j}^{\mu}-a_{j}^{\lambda} a_{i}^{\mu}\right),
\end{aligned}
$$

$\alpha=1, \ldots, s$. Since $\omega^{i} \wedge \omega^{j}, i<j$, are independent on $N(3.10)$ implies

$$
T_{i j}^{\alpha}+\sum_{\mu=n+1}^{p} T_{i \mu}^{\alpha} a_{j}^{\mu}-\sum_{\lambda=n+1}^{p} T_{j \lambda}^{\alpha} a_{i}^{\lambda}+\sum_{\substack{\lambda<\mu \\ \lambda, \mu=n+1, \ldots, p}} T_{\lambda \mu}^{\alpha}\left(a_{i}^{\lambda} a_{j}^{\mu}-a_{j}^{\lambda} a_{i}^{\mu}\right)=0
$$

for each $\alpha=1, \ldots, s$ and each pair $(i j)$ with $i<j, i, j=1, \ldots, n$. In matrices we write $(3.11)$ as

$$
\mathcal{T} A=0,
$$

where $A$ is a matrix of size $\left(\begin{array}{l}p \\ 2\end{array}\right) \times\left(\begin{array}{l}n \\ 2\end{array}\right)$ given as follows: for a pair $I=(i j)$ with $i<j, i, j=1, \ldots, n, I$-th column of $A$ is

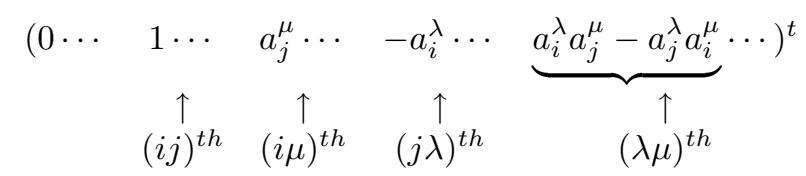


for $n<\lambda<\mu$. Observe that the first $\left(\begin{array}{l}n \\ 2\end{array}\right)$ rows or $A$ is the identity matrix, therefore $A$ is of maximal rank. In particular, if $n=p$, then $A$ is the identity matrix of size $\left(\begin{array}{l}p \\ 2\end{array}\right)$. Therefore, $\mathcal{T}$ is identically zero on an integral manifold of maximal dimension $p$.

Observe that (3.12) is a system of $\left(\begin{array}{l}n \\ 2\end{array}\right)$ independent linear equations on the $\left(\begin{array}{l}p \\ 2\end{array}\right)$ columns of $\mathcal{T}$. Hence we have:

Theorem 3.4. If $N$ is an integral manifold of (3.5) of dimension $n, 2 \leq n \leq p$, then the number of linearly independent columns of $\mathcal{T}$ is at most $\left(\begin{array}{l}p \\ 2\end{array}\right)-\left(\begin{array}{l}n \\ 2\end{array}\right)$.

Definition 3.5. Given a set of smooth functions $T_{\alpha}, \alpha=1, \ldots, k$ on $M$ a smooth function $\rho$ is said to be a common factor of $T_{\alpha}$ 's if $T_{\alpha}=\rho \phi_{\alpha}$, for some smooth function $\phi_{\alpha}$ for each $\alpha=1, \ldots, k$.

Theorem 3.6. Let $\theta^{1}, \ldots, \theta^{s}, \omega^{1}, \ldots, \omega^{p}$ be 1 -forms of $M^{m}, s+p=m$, as in (3.5)-(3.6). Let $n, 2 \leq n \leq p$, be an integer. Then there exists an integral manifold $N$ of (3.5) of dimension $n$ if and only if there exists a non-degenerate set of functions $\rho=\left(\rho_{1}, \ldots, \rho_{m-n}\right)$ having the following properties: on the common zero set of $\rho$ the first $\left(\begin{array}{l}n \\ 2\end{array}\right)$ columns $\mathcal{T}_{1}, \ldots, \mathcal{T}_{\left(\begin{array}{c}n \\ 2\end{array}\right)}$ belong to the linear span of $\mathcal{T}_{\lambda}, \lambda=\left(\begin{array}{c}n \\ 2\end{array}\right)+1, \ldots,\left(\begin{array}{l}p \\ 2\end{array}\right)$, on $N$, where $\mathcal{T}_{\lambda}$ is the $\lambda$-th column of $\mathcal{T}$, and

$$
\theta^{\alpha}=0 \bmod (\rho, d \rho) \text {. }
$$

Corollary 3.7. Under the same hypotheses as in Theorem 3.6 suppose that $s \geq\left(\begin{array}{l}p \\ 2\end{array}\right)-\left(\begin{array}{l}n \\ 2\end{array}\right)+1$. Then there exists an integral manifold of (3.5) of dimension $n$ if and only if there exists a non-degenerate set of real-valued functions $\rho=$ $\left(\rho_{1}, \ldots, \rho_{m-n}\right)$ such that the determinants of square submatrices of $\mathcal{T}$ of size $\left(\begin{array}{l}p \\ 2\end{array}\right)-\left(\begin{array}{l}n \\ 2\end{array}\right)+1$ are zero modulo $\rho$ and $\rho$ satisfies (3.13).

Corollary 3.8. Under the same hypotheses as in Theorem 3.6, if $s=1$, then there exists an integral manifold of dimension $n$ if and only if there exists a non-degenerate set of smooth functions $\rho=\left(\rho_{1}, \ldots, \rho_{m-n}\right)$ such that

$$
T_{i j}=0 \bmod \left(\rho, T_{\lambda \mu}: \text { either } \lambda>n \text { or } \mu>n\right)
$$

that satisfies (3.13).

Proposition 3.9. Suppose that a submanifold $N^{n} \subset M^{m}$ given as the common zero locus of a non-degenerate set of smooth real-valued functions $\rho=$ $\left(\rho_{1}, \ldots, \rho_{m-n}\right)$ is an integral manifold of (3.5) of dimension $n<p$. Then $N$ is contained in a (unique) integral manifold $\tilde{N}$ of maximal dimension $p$ if and only if there exists a non-degenerate set of smooth real-valued functions $\tau=\left(\tau_{1}, \ldots, \tau_{s}\right)$ with the following properties:

i) $\tau_{j} \equiv 0 \bmod \rho$ for each $j=1, \ldots, s$,

ii) $\theta^{\alpha} \equiv 0 \bmod (\tau, d \tau), \alpha=1, \ldots, s$.

$\rho_{1}, \ldots, \rho_{m-n}$ and $\tau_{1}, \ldots, \tau_{s}$ in the above propositions can be obtained from the factorization of the coefficients of $d \theta^{\alpha}$ : Let $\tau_{i j}^{\alpha}$ be the LHS of (3.11) for each 
$\alpha=1, \ldots, s, i, j=1, \ldots, n$, and let $T_{i j}^{\alpha}, \alpha=1, \ldots, s$ and $i, j=1, \ldots, p$ be as in (3.6). Then $\tau_{i j}^{\alpha}$ are zero modulo $\rho$ and $T_{i j}^{\alpha}$ are zero modulo $\tau$.

Remark. If each 1-form of (3.5)-(3.6) is real-analytic $\left(C^{\omega}\right)$, then $T_{i \bar{j}}^{\alpha}$ are $\left(C^{\omega}\right)$ and therefore, factorizable into a product of finitely many complex valued functions $f$ with $d f(P) \neq 0$. The factorization is unique modulo unit.

Now we are concerned with the problem of deciding whether $M$ is foliated by integral manifolds of dimension $n<p$. In the case $s=1$, i.e., the Pfaffian system (3.5) consists of a single 1-form $\theta$, is the classical Pfaff problem (see [1, Chapter II]). Let $\theta$ be a smooth 1 -form on a smooth manifold $M^{m}$. The rank $r$ is defined by the conditions

$$
\theta \wedge(d \theta)^{r} \neq 0, \quad \theta \wedge(d \theta)^{r+1}=0 .
$$

There is a second integer $t$ defined by

$$
(d \theta)^{t} \neq 0, \quad(d \theta)^{t+1}=0 .
$$

Elementary arguments show that there are two cases:

$$
\begin{aligned}
& \text { (i) } \quad t=r \text {; } \\
& \text { (ii) } t=r+1 \text {. }
\end{aligned}
$$

The first is the case $\theta \wedge(d \theta)^{r} \neq 0$ and $(d \theta)^{r+1}=0$ and the second is the case $(d \theta)^{r+1} \neq 0$ and $\theta \wedge(d \theta)^{r+1}=0$. The following theorem is due to Darboux.

Theorem 3.10. Let $\theta$ be a 1-form. In a neighborhood suppose $r$ and $t$ are constant. Then $\theta$ has the normal form

$$
\begin{aligned}
& \theta=y^{0} d y^{1}+\cdots+y^{2 r} d y^{2 r+1} \quad \text { if } r+1=t, \\
& \theta=d y^{1}+y^{2} d y^{3}+\cdots y^{2 r} d y^{2 r+1} \quad \text { if } r=t .
\end{aligned}
$$

In these expressions, the $y$ 's are independent functions and are therefore parts of a local coordinate system.

Proof. See [1] page 40.

Corollary 3.11. Suppose that $\theta$ is a smooth 1 -form of rank $r$ on $M^{m}$ and that $2 r+1 \leq m$. Then $M$ is foliated by integral manifolds of dimension $m-(r+1)$. Integral manifolds are given by $y_{2 k-1}=$ const, $k=1, \ldots, r+1$. In particular, if $\theta$ is of rank 0 , which is the case of the Frobenius integrability, then $M$ is foliated by integral manifolds of dimension $m-1$.

\section{$\S 4$. Some applications and problems in several complex variables}

In this section we present applications in several complex variables. 


\subsection{Complex submanifolds in real hypersurfaces}

Let $\mathbb{C}^{n+1}:=\{(z, w)\}$, where $z=\left(z_{1}, \ldots, z_{n}\right)$. Let $r$ be a non-degenerate real-valued function on a neighborhood $U$ of origin of $\mathbb{C}^{n+1}$. We consider a real hypersurface $M:=\left\{(z, w) \in \mathbb{C}^{n+1}: r(z, w)=0\right\}$. Problem is to find necessary and sufficient conditions in terms of derivatives of $r$ for there to exist a complex manifold of complex dimension $p=1, \ldots, n$. Let $\theta:=i \partial r$ (cf. [6]). Then the problem is finding integral manifold $N^{2 p}$ of $\theta=0$ which is invariant under the complex structure tensor $J$. This problem belongs to the second kind ( $\S 3$ ). Hence by Corollary 3.11, we find rank of $\theta$ first. $d \theta \bmod \theta$ is called the Leviform. The case of rank 0 is called the Levi-flat case. In [17] we solved this problem and presented several examples in $\mathbb{C}^{3}$ and $\mathbb{C}^{4}$.

\subsection{Complex submanifolds in almost complex manifolds}

Let $\left(M^{2 m}, J\right)$ be a smooth almost complex manifold. For a real tangent vector $X \in T M$ let $X^{\prime}=1 / 2(X-\sqrt{-1} J X)$ and $X^{\prime \prime}=1 / 2(X+\sqrt{-1} J X)$. The complex vectors $X^{\prime}$ and $X^{\prime \prime}$, which we shall call $(1,0)$ part of $X$ and $(0,1)$ part of $X$, respectively, are eigenvectors of $J$ associated with the eigenvalues $+i$, and $-i$, respectively. Then we have $X=X^{\prime}+X^{\prime \prime}$ and the decomposition of the complexified tangent bundle:

$$
T_{\mathbb{C}} M=T^{\prime} M \oplus T^{\prime \prime} M,
$$

where $T^{\prime} M$ and $T^{\prime \prime} M$ are the set of all $(1,0)$ vectors and $(0,1)$ vectors, respectively. Then we see that $\overline{T^{\prime} M}=T^{\prime \prime} M$. On a neighborhood of the reference point $P \in M$ let $L_{1}, \ldots, L_{m}$ and $\bar{L}_{1}, \ldots, \bar{L}_{m}$ be smooth sections of $T^{\prime} M$ and $T^{\prime \prime} M$, respectively, that are linearly independent at every point. Let $\theta^{1}, \ldots, \theta^{m}, \bar{\theta}^{1}, \ldots, \bar{\theta}^{m}$ be the dual 1-forms. For a smooth function $t$ we define $\partial t:=\sum_{j=1}^{m}\left(L_{j} t\right) \theta^{j}$ and $\bar{\partial} t:=\sum_{j=1}^{m}\left(\bar{L}_{j} t\right) \bar{\theta}^{j}$. Then we see that $d t=\partial t+\bar{\partial} t$. A submanifold $N^{2 n} \hookrightarrow M$ is a complex submanifold if and only if $N$ is an integral manifold of the complex Pfaffian system $\bar{\theta}:=\left(\bar{\theta}^{1}, \ldots, \bar{\theta}^{m}\right)$. Thus we set

$$
d \bar{\theta}^{\lambda} \equiv \sum_{\mu<\nu} T_{\mu \nu}^{\lambda} \theta^{\mu} \wedge \theta^{\nu} \quad \bmod \bar{\theta},
$$

where $\lambda, \mu, \nu=1, \ldots, m$. The RHS of (4.1) is basically same as the Nijenhuis tensor, which is the obstruction to the integrability. In [15] we discuss the existence of integral manifolds using a complexified version of our theory of $\S 3$.

\subsection{Problem: Minimality and maximality of real submanifolds}

Let $M \subset \mathbb{C}^{n+d}, n \geq 0$, be a $C^{\infty}$ real hypersurface defined as a common zero locus of $d$ nondegenerate real-valued function $r_{1}, \ldots, r_{d}, d \geq 2$. The CR structure bundle of $M$ is $H(M):=T(M) \cap J T(M)$, which is the subbundle of the tangent bundle of maximal complex subspaces. The standard complex structure on $\mathbb{C}^{n+d}$ gives the decomposition $H_{\mathbb{C}}(M):=H^{\prime}(M) \oplus H^{\prime \prime}(M)$. Let

$$
\theta^{j}:=\sqrt{-1} \partial r_{j}, \quad j=1, \ldots, d .
$$


$M$ is said not to be minimal (cf. [23]) if there exists a submanifold $M^{\prime} \subset M$ with the same CR structure bundle, that is, $H\left(M^{\prime}\right)=H(M)$. Then the problem is whether one can tell by the derivatives of the defining functions $r_{1}, \ldots, r_{d}$ whether or not $M$ is minimal. There is an analogous problem on maximality. As typical examples, the intersection of the sphere $\sum_{j=1}^{n}\left|z_{j}\right|^{2}=1$ with a complex submanifold is a real submanifold of maximal CR structure. If $M$ is a real submanifold of codimension $d$, with the complex dimension of $H^{\prime}(M)$ is $(n+d)-k$, then $d / 2 \leq k \leq d$. Maximality is the cases $k=d / 2$ if $d$ is even and $k=(d+1) / 2$ if $d$ is odd.

\subsection{Problem: Invariant submanifolds of almost complex}

In $\mathbb{C}^{n}$, we consider the complex 1 -forms

$$
\theta^{j}:=d z^{j}+\sum_{k=1}^{n} A_{k}^{j}(z, \bar{z}) d \bar{z}^{k}, \quad j=1, \ldots, n,
$$

where $A_{k}^{j}$ are smooth functions that vanish at the origin. Let $J$ be the almost complex structure whose $(1,0)$ forms are given by $\theta:=\left(\theta^{1}, \ldots, \theta^{n}\right)$, called the purturbation of the standard complex structure by $A_{k}^{j}$. Then the problem is finding conditions on $A_{k}^{j}$ for there to exist a $J$-invariant submanifold of $M$.

\section{References}

[1] R. Bryant, S. S. Chern, R. Gardner, H. Goldschmidt, and P. Griffiths, Exterior Differential Systems, Springer-Verlag, New York, 1986.

[2] R. Bryant, Exterior differential system, Lectures at Duke Univ. noted by Sungho Wang.

[3] E. Cartan, Leçons sur les invariants intégraux, Hermann, Paris, 1922.

[4] L Les systèmes différentiels extérieurs et leurs applications, géométriques Hermann, 1971 Photocopy Paris, 1945.

[5] J. S. Cho and C. K. Han, Complete prolongation and the Frobenius integrability for overdetermined systems of partial differential equations, J. Korean Math. Soc. 39 (2002), no. 2, 237-252.

[6] S. S. Chern and J. K. Moser, Real hypersurfaces in complex manifolds, Acta Math. 133 (1974), 219-271.

[7] A. Clebsch, Über die simultane Integration linearer partieller Differentialgleichungen, J. Reine und Angew. Math. (Crelle) 65 (1866), 257-268.

[8] G. Darboux, Sur le problème de Pfaff (1), (2), Bull. Sci. Math. 6 (1882), 14-36, 49-68.

[9] F. Deahna, Über die Bedingungen der Integrabilitat, J. Reine und Angew. Math. 20 (1840), 340-350.

[10] G. Frobenius, Über das Pfaffsche probleme, J. Reine und Angew. Math. 82 (1877), 230-315.

[11] P. Griffiths and G. Jensen, Differential Systems and Isometric Embeddings, Annals of Mathematics Studies, 114. The William H. Roever Lectures in Geometry. Princeton University Press, Princeton, NJ, 1987.

[12] C. K. Han, Solvability of overdetermined PDE systems that admit a complete prolongation and some local problems in CR geometry, J. Korean Math. Soc. 40 (2003), no. 4, 695-708. 
[13] _ Pfaffian systems of Frobenius type and solvability of generic overdetermined PDE systems, Symmetries and overdetermined systems of partial differential equations, 421-429, IMA Vol. Math. Appl., 144, Springer, New York, 2008.

[14] _ Overdetermined PDE systems of generic type, RIM Lecturenote Series 53, Seoul National U., 2007.

[15] C. K. Han and K. H. Lee, Integrable submanifolds in almost complex submanifolds, preprint.

[16] C. K. Han, J. W. Oh, and G. Schmalz, Symmetry algebra for integral curves of $2 n$ vector fields on $(2 n+1)$-manifold, Math. Ann. 341 (2008), 529-542.

[17] C. K. Han and G. Tomassini, Complex submanifolds in real hypersurfaces, preprint.

[18] M. Kuranishi, On E. Cartan's prolongation theorem of exterior differential systems, Amer. J. Math. 79 (1957), 1-47.

[19] H. B. Lawson, The quantitative theory of foliations, Expository lectures from the CBMS Regional Conference held at Washington University, St. Louis, Mo., January 6-10, 1975. Conference Board of the Mathematical Sciences Regional Conference Series in Mathematics, No. 27. American Mathematical Society, Providence, R. I., 1977.

[20] A. Newlander and L. Nirenberg, Complex analytic coordinates in almost complex manifolds, Ann. of Math. (2) 65 (1957), 391-404.

[21] A. Nijenhuis and W. B. Woolf, Some integration problems in almost-complex and complex manifolds, Ann. of Math. (2) 77 (1963), 424-489.

[22] J. M. Trépreau, Sur le prolongement holomorphe des fonctions $C-R$ défines sur une hypersurface réelle de classe $C^{2}$ dans $C^{n}$, Invent. Math. 83 (1986), no. 3, 583-592.

[23] A. Tumanov, Extension of CR-functions into a wedge, Mat. Sb. 181 (1990), no. 7, 951-964; translation in Math. USSR-Sb. 70 (1991), no. 2, 385-398.

[24] S. Wang, Exterior differential system, Lectures at Seoul Nat. Univ. based on R. Bryant's lectures at Duke Univ., 2005.

[25] F. W. Warner, Foundations of Differentiable Manifolds and Lie Groups, Scott, Foresman and Co., Glenview IL, 1971.

Department of Mathematics

Seoul National University

SEOUL 151-742, KoreA

E-mail address: ckhan@math.snu.ac.kr 